\title{
A Modified Selective Medium Containing Benzalkonium Chloride (BKC) for the Isolation of Pseudomonas aeruginosa from Raw Milk
}

\author{
Aylin Akoglu ${ }^{1 *}$, Evrim Gunes Altuntas ${ }^{2}$, Gokce Polat Yemis ${ }^{1}$ \\ ${ }^{1}$ Department of Food Engineering, Faculty of Engineering, University of Ankara, Ankara, Turkey; ${ }^{2}$ Institute of Biotechnology, Uni- \\ versity of Ankara, Ankara, Turkey. \\ Email: "aylinsen61@yahoo.com
}

Received January $31^{\text {st }}, 2012$; revised June $12^{\text {th }}, 2012$; accepted June $19^{\text {th }}, 2012$

\begin{abstract}
A modified selective medium (modified Cetrimide Agar, mCA) consisting of $200 \mu \mathrm{g} / \mathrm{mL}$ benzalkonium chloride (BKC) was developed for the isolation of Pseudomonas aeruginosa from raw milk. Initially, a total of 55 isolates were obtained from 14 raw milk samples collected from several dairy plants in Ankara, Turkey. Among these isolates, 19 were identified as Pseudomonas aeruginosa, 28 as Pseudomonas fluorescens, 4 as Acinetobacter baumannii, 2 as Enterobacter intermedium, 1 as Enterobacter agglomerans, and 1 as Escherichia coli using Microbact biochemical test kit. $\mathrm{BKC}$ was chosen as a selective agent to suppress growth of competitive flora because it is very effective against a wide range of Gram-negative bacteria while P. aeruginosa is resistant. MICs (minimum inhibitory concentration) for BKC were determined by agar dilution method. The concentration of $200 \mu \mathrm{g} / \mathrm{mL}$ BKC inhibited competitive flora, while $90 \%$ of $P$. aeruginosa strains were resistant. When the results of enumeration of $P$. aeruginosa and other Gram (-) bacteria in Cetrimide Agar (CA) and mCA were compared, it was observed that mCA was more selective than the standard CA in preventing the growth of competitive flora especially of $P$. fluorescens.
\end{abstract}

Keywords: P. aeruginosa; Benzalkonium Chloride (BKC); Modified Cetrimide Agar; Raw Milk

\section{Introduction}

Psychrotrophic bacteria, of which Pseudomonas is an important genus, are considered the most important pathogens contributing to milk spoilage through the production of lipolytic and proteolytic enzymes [1,2]. Many of these enzymes are not inactivated by pasteurizing at $72^{\circ} \mathrm{C}$ for $15 \mathrm{~s}$ or by Ultra-High Temperature (UHT) treatment. The residual activities of these enzymes can reduce the organoleptic quality and shelf life of processed milk products [3]. Gram-negative bacteria usually account for more than $90 \%$ of the microbial population in cold raw milk and among these bacteria Pseudomonas are the predominant microflora [4].

Although several selective media for the detection of Pseudomonas species are produced commercially, they lack specificity [1]. Conventional methods for the detection of $P$. aeruginosa use cetrimide as a selective agent. Cetrimide Agar and Pseudosel Agar (BBL) are devised to serve as selective media for the isolation and identification of Pseudomonas aeruginosa while supposedly inhibiting the growth of other microorganisms $[5,6]$. The

"Corresponding author.
$0.03 \%$ Cetrimide Agar is more selective for growth of $P$. aeruginosa than is Pseudosel Agar; however, certain bacteria other than $P$. aeruginosa also grow on the former medium. Although Pseudosel Agar is not a highly selective medium for $P$. aeruginosa, it is preferable to Technicolor Agar for detection of the pyocyanin and pyorubin pigments produced by $P$. aeruginosa [5]. Therefore, the performance of available culture method is of great importance. Most frequently, Cetrimide Broth or Cetrimide Agar or MacConkey Agar are used [7]. Although Cetrimide Agar is strongly selective for $P$. aeruginosa, some strains of Gram-negative bacilli (especially Klebsiella spp. and Providencia) can grow on it [8]. The potent inhibitory substances in these media not only suppress the competing bacterial flora but may also exert an adverse effect on the targeted bacterium [9].

There is an increasing concern that the use of biocides such as benzalkonium chloride (BKC) [10]. BKC has a broad-spectrum antimicrobial activity $[10,11]$. Most of $P$. aeruginosa strains resistant and adapted to BKC also results in higher resistance to amphoteric disinfectants [12].

The aim of the current study was to develop an improved selective Cetrimide Agar for the isolation of 
Pseudomonas aeruginosa from raw milk. BKC was chosen as a selective agent for the development of a modified selective Agar, because BKC has an important effect on variety of Pseudomoas species and other pathogens which are able to grow on Cetrimide Agar and does not have an inhibitory effect on Pseudomonas aeruginosa. In this study, most acceptable concentration of BKC was tried to detect to make Cetrimide Agar base more selective for Pseudomonas aeruginosa.

\section{Materials and Methods}

\subsection{Microorganisms and Media}

Pseudomonas aeruginosa ATCC 29212 was used as a reference strain for this study. Other bacterial strains were isolated from raw milk samples collected from several dairy plants in Turkey. Cetrimide Agar (CA) used for isolation was prepared from dehydrated powders in accordance with manufacturer's instructions (Merck, Darmstadt, Germany). In order to prepare modified CA (mCA) plates, $\mathrm{CA}$ at $50^{\circ} \mathrm{C}$ was supplemented with $\mathrm{BKC}$ which was used as a selective agent to improve selectivity of CA. In addition to CA medium, Tryptic Soy Agar (TSA, Merck, Darmstadt, Germany) was used as the control medium.

\subsection{Isolation and Identification}

Raw milk samples that were carried to laboratory at $4^{\circ} \mathrm{C}$ were plated on Cetrimide Agar by spread plate technique and the plates were incubated at $28^{\circ} \mathrm{C}$ for $72 \mathrm{~h}$. The colonies isolated on these plates were identified using Microbact 24E Gram negative Identification System (Oxoid, Cambridge, UK). Stock cultures were maintained at $-21^{\circ} \mathrm{C}$ in Tryptic Soy Broth (TSB, Merck, Darmstadt, Germany) containing $20 \%(\mathrm{v} / \mathrm{v})$ glycerol.

\subsection{Determination of Minimum Inhibitory Concentrations (MICs) for BKC}

MICs for BKC were determined by Agar dilution method. MICs were performed with various concentrations of BKC $(50,100,150,200,250,300,350,400,450,500$, $550,600 \mu \mathrm{g} / \mathrm{mL}$ ) in Cetrimide Agar. P. aeruginosa and other Gram (-) bacteria were grown at $18 \mathrm{~h}$ on TSB and 2 $\mu l$ aliquot of each strain was inoculated onto each of the Agar plates, then the plates were incubated at $37^{\circ} \mathrm{C}$ overnight. The lowest concentration preventing growth of cultures was taken to be the MIC [13]. This experiment was performed in duplicate.

\section{Results and Discussion}

\subsection{Isolation and Identification}

Many selective agents such as cetrimide, cephaloridine, fucidin nalidixic acid, phenanthroline, Irgasan have been used to obtain selective media for isolation and identification of Pseudomonas aeruginosa. Although several selective media for $P$. aeruginosa have been described, in fact none of them is absolutely specific [13]. Nowadays, Cetrimide Agar is commonly used for isolation and identification of Pseudomonas aeruginosa; however studies indicate that some strains of Gram-negative bacilli can grow on it [8].

A total of 14 raw milk samples were examined for isolation of $P$. aeruginosa and other Gram (-) bacteria using Cetrimide Agar and a total of 55 isolates were obtained and identified. Pseudomonas spp. accounted for $85.5 \%$ of the total isolates and among these isolates $P$. aeruginosa and $P$. fluorescens accounted for $34.6 \%$ and $50.9 \%$, respectively.

The main psychrotrophic microflora encountered in raw milk are Gram-negative rods composed mainly of psychrotrophic species of Pseudomonas, Achromobacter, Aeromonas, Serratia, Alcaligenes, Chromobacterium, Flavobacterium and Enterobacter. In milk, Pseudomonas spp. comprise at least $50 \%$ of the total bacteria [3]. In the current study, Pseudomonas was the most predominant genus, and $P$. fluorescens was the most predominant species isolated from raw milk samples. In addition to these bacteria, Acinetobacter baumannii, Enterobacter intermedium, Enterobacter agglomerans and Escherichia coli accounted for $7.2 \%, 3.6 \%, 1.8 \%$ and $1.8 \%$ of the 55 isolates, respectively (Table 1). It is known that the predominant flora of the raw milk is $P$. fluorescens. Ternström et al. [14] observed that $P$. fluorescens was present in $84 \%$ of the raw milk samples. Similarly, Gennarl and Dragotto [15] showed that $P$. fluorescens accounted for $55.6 \%$ of the all bacterial isolates in raw milk. Hence, there is a need to inhibit these other Gram (-) bacteria especially $P$. fluorescens, so as to enhance selectivity of CA for $P$. aeruginosa.

\subsection{MICs for BKC}

Benzalkonium chloride is a nitrogen-based quaternary

Table 1. Total number of Pseudomonas aeruginosa and other Gram-negative bacteria isolated from raw milk samples on Cetrimide Agar.

\begin{tabular}{lcc}
\hline \multicolumn{1}{c}{ Bacteria strains } & Total (55) & Percent of total isolates (\%) \\
\hline Pseudomonas aeruginosa & 19 & 34.6 \\
Pseudomonas fluorescence & 28 & 50.9 \\
Enterobacter agglomerans & 1 & 1.8 \\
Acinetobacter baumannii & 4 & 7.2 \\
Escherichia coli & 1 & 1.8 \\
Enterobacter intermedium & 2 & 3.6 \\
\hline
\end{tabular}


ammonium compound (QAC) demonstrating broad-spectrum antimicrobial activity [12]. It is well known that Gram-negative bacteria are less susceptible to QACs than Gram-positive bacteria, and Pseudomonas spp. have generally high intrinsic resistance compared with other Gram-negative bacteria. P. aeruginosa is often used in disinfectant testing because of its intrinsically high resistance to disinfectants [16] $P$. aeruginosa may adapt to high concentrations of QACs. The resistance mechanisms involved in the adaptation process is not fully understood [12].

Study of MICs for BKC demonstrated that $P$. aeruginosa strains were able to grow at a concentration of 500 $\mu \mathrm{g} / \mathrm{mL}$ BKC while only two isolates $(10 \%$ of total $P$. aeruginosa) were inhibited at a concentration of 300 $\mu \mathrm{g} / \mathrm{mL}$. The entire competitive flora was suppressed by $<200 \mu \mathrm{g} / \mathrm{mL}$ BKC. Since two of the P. aeruginosa strains

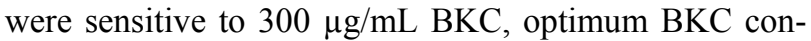
centration was determined as $200 \mu \mathrm{g} / \mathrm{mL}$ to retain two $P$. aeruginosa (Table 2). These results showed that $P$. aeruginosa was more resistant to $\mathrm{BKC}$ than $P$. fluorescens and other Gram negative bacteria. Abraham et al. [17] informed that $P$. aeruginosa was resistant to some antimicrobial agents, as we observed.

\subsection{Comparison of Selectivity of Two Media}

Enumeration of $P$. aeruginosa and other Gram (-) bacteria were performed in $\mathrm{CA}$ and $\mathrm{mCA}$ and the results were shown in Table 3. $200 \mu \mathrm{g} / \mathrm{mL}$ BKC was added to CA to suppress the growth of competitive flora. After incubation, while the counts of $P$. aeruginosa strains remained constant with $0.68 \log$ reduction, only two $P$. aeruginosa strains were determined sensitive to BKC and counts to be decreased by $5.4 \mathrm{log}$. In addition, the count of P. fluorescens strains were reduced $5.57 \mathrm{log}$. Counts of Acinetobacter baumannii, Enterobacter intermedium, Enterobacter agglomerans and Escherichia coli decreased 4.41, 4.77, 5.68, 7.10 log, respectively. These results indicated that $200 \mu \mathrm{g} / \mathrm{mL}$ BKC concentrations could inhibit of

Table 2. Minimum Inhibition Concentrations (MICs) for BKC.

\begin{tabular}{lc}
\hline \multicolumn{1}{c}{ Strains } & MIC for BKC $(\boldsymbol{\mu g} / \mathbf{m L})$ \\
\hline P. aeruginosa ATCC 29212 & 500 \\
P. aeruginosa (17 strains) & 500 \\
P. aeruginosa (2 strains) & 300 \\
P. fluorescence (28 strains) & 150 \\
E. agglomerans (1 strain) & 100 \\
A. baumannii (4 strains) & 150 \\
E. coli (1 strain) & $<50$ \\
E. intermedium (2 strains) & 100 \\
\hline
\end{tabular}

Table 3. Enumeration of $P$. aeruginosa and competitive flora in CA and $\mathrm{mCA}(\log \mathrm{cfu} / \mathrm{mL})^{*}$.

\begin{tabular}{lccc}
\hline \multicolumn{1}{c}{ Strains } & CA & mCA & Reduction \\
\hline P. aeruginosa ATCC 29212 & $8.79 \pm 0.52$ & $8.03 \pm 0.95$ & 0.76 \\
P. aeruginosa (17) & $8.62 \pm 0.41$ & $7.93 \pm 0.43$ & 0.69 \\
P. aeruginosa (2) & $8.78 \pm 0.78$ & $3.38 \pm 0.23$ & 5.40 \\
P. fluorescence (28) & $8.54 \pm 0.71$ & $2.98 \pm 0.26$ & 5.56 \\
E. agglomerans (1) & $8.28 \pm 0.98$ & $3.52 \pm 0.31$ & 4.76 \\
A. baumannii (4) & $7.86 \pm 0.87$ & $3.44 \pm 0.30$ & 4.42 \\
E. coli (1) & $8.10 \pm 0.34$ & $<1.00$ & 7.10 \\
E. intermedium (2) & $8.38 \pm 0.54$ & $2.70 \pm 0.22$ & 5.68 \\
\hline
\end{tabular}

*The results of enumerations were calculated by the average of each related strains.

competitive flora significantly, whereas $90 \%$ of all $P$. aeruginosa strains was not affected. When the two media were compared, the modified Cetrimide Agar was more selective than the standard CA for P. aeruginosa.

\section{Conclusion}

Selective media include Nutrient Agar supplemented with antibiotics, Cetrimide Agar, Pseudomonas Isolation Agar and growth media supplemented with C-390 (9chloro-9-[4-(diethylamino)phenyl]-9,10-dihydro-10-phen ylacridine hydrochloride) or with phenanthroline have been used for isolation of $P$. aeruginosa. But none of these supplements were a perfect selective agent. Some selective agents suppressed growth of Gram (-) bacteria as well as the growth of P. aeruginosa. To obviate this potential problem, in the current study BKC was used at a concentration of $200 \mu \mathrm{g} / \mathrm{mL}$ which suppressed the growth of other Gram-negative bacteria but does not have an effect on normal growth of $P$. aeruginosa. Our results showed that BKC modification to the Cetrimide Agar improved its selectivity by preventing the growth of other Gram-negative bacteria. This modified medium should be convenient medium to isolate of $P$. aeruginosa from raw milk.

\section{Acknowledgements}

The authors would like to thank Prof. Dr. A. Kadir Halkman to support this study.

\section{REFERENCES}

[1] S. Flint and N. Hartley, "A Modified Selective Medium for the Detection of Pseudomonas Species that Cause Spoilage of Milk and Dairy Products," International Dairy Journal, Vol. 6, No. 2, 1996, pp. 223-230. doi:10.1016/0958-6946(95)00007-0

[2] T. S. Gunasekera, M. R. Dorsch, M. B. Slade and D. A. 
Veal, "Specific Detection of Pseudomonas spp. in Milk by Fluorescence in Situ Hybridization Using Ribosomal RNA Directed Probes," Journal of Applied Microbiology, Vol. 94, No. 5, 2003, pp. 936-945. doi:10.1046/j.1365-2672.2003.01930.x

[3] M. L. Martins, C. L. O. Pinto, R. B. Rocha, E. F. Araújo and M. C. D. Vanetti, "Genetic Diversity of Gram-Negative, Proteolytic, Psychrotrophic Bacteria Isolated from Refrigerated Raw Milk," International Journal of Food Microbiology, Vol. 111, No. 2, 2006, pp. 144-148. doi:10.1016/j.ijfoodmicro.2006.06.020

[4] B. Doğan and K. J. Boor, "Genetic Diversity and Spoilage Potentials among Pseudomonas spp. Isolated from Fluid Milk Products and Dairy Processing Plants," Applied and Environmental Microbiology, Vol. 69, No. 1, 2003, pp. 130-138. doi:10.1128/AEM.69.1.130-138.2003

[5] W. Dwight, J. R. Lambe and P. Stewart, "Evaluation of Pseudosel Agar as an Aid in the Identification of Pseudomonas aeruginosa," Applied Microbiology, Vol. 23, No. 2, 1972, pp. 377-381.

[6] R. Ramalho, J. Cunha, P. Teixeira and P. A. Gibbs, "Modified Pseudomonas Agar: New Differential Medium For the Detection/Enumeration of Pseudomonas aeruginosa in Mineral Water," Journal of Microbiology Methods, Vol. 49, No. 1, 2002, pp. 69-74. doi:10.1016/S0167-7012(01)00365-7

[7] L. Laine, J. D. Perry, J. Lee, M. Oliver, A. L. James, C. De La Foata, D. Halimi, S. Orenga, A. Galloway and F. K. Gould, "A Novel Chromogenic Medium for Isolation of Pseudomonas aeruginosa from the Sputa of Cystic Fibrosis Patients," Journal of Cystic Fibrosis, Vol. 8, No. 2, 2009, pp. 143-149. doi:10.1016/j.jcf.2008.11.003

[8] H. A. Lilly and E. J. L. Lowbury, "Cetrimide-Nalidixic Acid Agar as a Selective Medium for Pseudomonas aeruginosa," Journal of Medical Microbiology, Vol. 5, No. 1, 1972, pp. 151-153. doi:10.1099/00222615-5-1-151

[9] G. Szita, V. Tabajdi, A. Fabian, G. Biro, O. Reichard and P. S. Körmöczy, “A Novel, Selective Synthetic Acetamide Containing Culture Medium for Isolating Pseudomonas aeruginosa from Milk," International Journal of Food Microbiology, Vol. 43, No. 1-2, 1998, pp. 123-127. doi:10.1016/S0168-1605(98)00104-4

[10] P. H. McCay, A. A. Ocampo-Sosa and G. T. A. Fleming,
"Effect of Subinhibitory Concentrations of Benzalkonium Chloride on the Competitiveness of Pseudomonas aeruginosa Grown in Continuous Culture," Microbiology, Vol. 156, No. 1, 2010, pp. 30-38. doi:10.1099/mic.0.029751-0

[11] K. Nagai, S. Ohta, H. Zenda, H. Matsumoto and M. Makino, "Biochemical Characterization of a Pseudomonas fluorescens Strain Isolated from a Benzalkonium Chloride Solution," Biological and Pharmaceutical Bulletin, Vol. 19, No. 6, 1996, pp. 873-875. doi: $10.1248 / \mathrm{bpb} .19 .873$

[12] S. Langsrud, G. Sundheim and R. Borgmann-Strahsen, "Intrinsic and Acquired Resistance to Quaternary Ammonium Compounds in Food-Related Pdeudomonas spp.," Journal of Applied Microbiology, Vol. 95, No. 4, 2003 pp. 874-882. doi:10.1046/j.1365-2672.2003.02064.x

[13] M. E. Campbell, S. W. Farmer and D. P. Speert, "New Selective Medium for Pseudomonas aeruginosa with Phenanthroline and 9-Chloro-9-[4-(diethyamino) phenyl]9,10-dihydro-10-phenylacridine Hydrochloride (C-390)," Journal of Clinical Microbiology, Vol. 26, 1988, pp. 19101912.

[14] A. A. Ternström, M. Lindberg and G. Molin, "Classification of the Spoilage Flora of Raw and Pasteurized Bovine Milk, with Special Reference to Pseudomonas and Bacillus," Journal of Applied Bacteriology, Vol. 75, No. 1, 1993, pp. 25-34. doi:10.1111/j.1365-2672.1993.tb03403.x

[15] M. Gennarl and F. Dragotto, "A Study of the Incidence of Different Fluorescent Pseudomonas Species and Biovars in the Microflora of Fresh and Spoiled Meat and Fish, Raw Milk, Cheese, Soil and Water," Journal of Applied Bacteriology, Vol. 72, No. 4, 1992, pp. 281-288. doi:10.1111/j.1365-2672.1992.tb01836.x

[16] A. D. Russell and I. Chopra, "Understanding Antibacterial Action and Resistance," 2nd Edition, Ellis Horwood, Hertfordshire, 1996.

[17] M. Abraham, P. Venter, J. F. R. Lues, I. Ivanov and O. Smidt, "Influence of Selected Antimicrobials on the Viability, Endotoxicity and Lipopolysaccharide Composition of Pseudomonas aeruginosa in Vitro," International Journal of Antimicrobial Agents, Vol. 34, No. 5, 2009, pp. 419-423. doi:10.1016/j.ijantimicag.2009.06.017 